\title{
Clinical characteristics and treatment of 69 patients with extranodal natural killer T-cell lymphoma
}

\author{
YUYAN GUO ${ }^{1}$, ENXIAO LI $^{2}$, LIPING SONG ${ }^{1 *}$ and YINYING WU ${ }^{2 *}$ \\ Departments of ${ }^{1}$ Radiotherapy Oncology and ${ }^{2}$ Oncology, \\ The First Affiliated Hospital of Xi'an Jiaotong University, Xi'an, Shaan'xi 710061, P.R. China
}

Received March 26, 2016; Accepted September 23, 2016

DOI: $10.3892 / \operatorname{mco} .2016 .1067$

\begin{abstract}
This study was conducted to retrospectively analyze the clinical characteristics and survival of patients with extranodal natural killer-T cell lymphoma (ENKL) and compare different treatment regimens. The univariate analysis demonstrated that survival was worse for patients with extranasal primary tumors, Eastern Cooperative Oncology Group performance status (ECOG PS) scores of $\geq 2$, International Prognostic Index (IPI) scores of 3-5, Ann Arbor stage III and IV disease, B symptoms, lymph node involvement and absence of radiotherapy. The Cox analysis demonstrated that ECOG PS score, stage, IPI, presence of B symptoms and radiotherapy were independent prognostic factors for overall survival (OS). The effect of diverse therapies on survival was then analyzed, and it was found that the 5-year survival rate of patients receiving chemoradiotherapy differed significantly from that of patients who received chemotherapy alone [hazard ratio $(\mathrm{HR})=0.347, \mathrm{P}=0.0203$ ], but did not differ significantly from the survival of patients treated with radiotherapy alone $(\mathrm{HR}=1.534, \mathrm{P}=0.6371)$. A subgroup analysis revealed a difference between the radiotherapy and non-radiotherapy groups for patients with extranasal-type stage III/IV disease. It was concluded that ECOG score, stage, IPI, presence of B symptoms and radiotherapy were independent prognostic factors for OS in patients with ENKL. The addition of radiotherapy achieved better outcomes compared with chemotherapy alone,
\end{abstract}

Correspondence to: Professor Liping Song, Department of Radiotherapy Oncology, The First Affiliated Hospital of Xi'an Jiaotong University, 277 Yanta West Road, Xi'an, Shaan'xi 710061, P.R. China

E-mail: xaslp@126.com

Dr Yinying Wu, Department of Oncology, The First Affiliated Hospital of Xi'an Jiaotong University, 277 Yanta West Road, Xi'an, Shaan'xi 710061, P.R. China

E-mail: shadowless_111@163.com

\section{"Contributed equally}

Key words: extranodal natural killer T-cell, lymphoma, clinical characteristics, prognosis, treatment but no difference was observed between chemoradiotherapy and radiotherapy. Patients with advanced-stage disease may also benefit from radiotherapy.

\section{Introduction}

Extranodal natural killer-T cell lymphoma (ENKL) is a subtype of non-Hodgkin lymphoma (1), occurring more frequently in Asia compared with Western countries (2). According to a recent survey, ENKL is the second most common extranodal lymphoma subtype in China after large B-cell lymphoma (3). ENKL is very aggressive, characteristically damaging vessels and surrounding tissues and initiating cytotoxic responses. The mean incidence is in middle age (43.1 years) (3). Over half of patients present with local symptoms, and the nasal region is the most common primary site (4). ENKL may also involve the skin, gastrointestinal tract, lung and liver, while soft tissue involvement is rare (5). The majority of the patients have B symptoms, whereas bone marrow involvement is rare (4). Patients typically have evidence of Epstein-Barr virus infection (6).

There is a range of reported treatments for ENKL, with varied efficacy. A Chinese report of 58 patients with early-stage disease (7) indicated that primary radiotherapy may increase the progression-free survival and 3-year overall survival. Chauchet et al (8) analyzed the optimal therapeutic options and factors affecting prognosis in 36 European patients with ENKL and concluded that early radiotherapy, concomitantly or sequentially with chemotherapy, may improve patient outcomes. However, Ma et al (9), in agreement with a recent meta-analysis (10), reported that the 5-year survival rates were not different between the radiotherapy and chemoradiotherapy groups in their study of 64 patients with early-stage nasal-type ENKL (57.9 and $61.5 \%$ respectively, $\mathrm{P}=0.47)$. The 2015 National Comprehensive Cancer Network (NCCN) guidelines (11) recommend several treatment regimens for ENKL for patients with different clinical characteristics. Chemotherapy and radiotherapy are currently the main options, but their efficacy remains controversial among different studies and in different countries.

The present study was conducted to retrospectively analyze the clinical characteristics and compare different treatment regimens for ENKL by investigating the survival of 69 patients with this disease, with the aim to summarize the experience of 
treating ENKL in clinical practice and provide a reference for future clinical treatment.

\section{Patients and methods}

Inclusion criteria. The inclusion criteria of our study were as follows: Pathologically confirmed ENKL with complete pathological data and absence of comorbid conditions. Follow-up data were complete, including physical examination, laboratory and imaging results, treatments administered and dates of recurrence and death.

Objective. This retrospective study was conducted at The First Affiliated Hospital of the Medical College of Xi'an Jiaotong University (Xi'an, China), and included 69 ENKL patients who were clinicopathologically diagnosed between 2004 and 2015 . Pathological diagnosis was made according to the World Health Organization classification criteria (12). Pathological data were collected from the Pathology Department of our hospital. The certain risk factors of ENKL were evaluated according to the 2015 NCCN guidelines, along with the International Prognostic Index (IPI), including age, serum lactate dehydrogenase (LDH), B symptoms, lymph node status, Ann Arbor stage, Eastern Cooperative Oncology Group performance status (ECOG PS) and number of extranodal sites.

Statistical analysis. Statistical analyses were performed using SPSS 21.0 statistical software (IBM Corp., Armonk, NY, USA) and GraphPad Prism software (GraphPad Software, San Diego, CA, USA). Clinical factors were estimated using Kaplan-Meier analysis and the log-rank test was used to assess significance of survival. The Chi-square test was used to compare the clinical factors between the radiotherapy and non-radiotherapy groups. Cox regression models were used for multivariate survival analysis. P-values $<0.05$ were considered to indicate statistically significant differences.

\section{Results}

Clinical characteristics and treatment regimens. The study included 50 men and 19 women, and 17 patients were aged $\geq 60$ years at diagnosis. The nasal cavity was the most common primary site $(58.0 \%)$. A total of 15 patients $(21.7 \%)$ had ECOG PS scores $\geq 2$, and 14 patients $(20.3 \%)$ had IPI scores of 3-5. A total of $44(63.8 \%)$ and $25(36.2 \%)$ patients had Ann Arbor stage I/II and III/IV disease, respectively. B symptoms were present at diagnosis in 33 patients $(47.8 \%)$, and 33 patients also had lymph node involvement, while only 3 patients had bone marrow involvement. The LDH levels were increased in 33 patients.

In our study, 5 patients underwent surgery, 7 underwent radiotherapy, 30 received chemotherapy alone and 29 received chemoradiotherapy. Chemotherapy combined with autologous hematopoietic stem cell transplantation (HSCT) was administered to 2 patients. In addition, 3 patients only received palliative care due to their poor health status. The initial chemotherapy regimens included cyclophosphamide, adriamycin, vincristine and prednisone (CHOP) in 36 cases, etoposide $+\mathrm{CHOP}$ (ECHOP) in 6 cases, gemcitabine + platinum (GP) in 4 cases, dexamethasone, methotrexate, ifosfamide, L-asparaginase and etoposide (SMILE) in 4 cases, and other regimens (e.g., L-asparaginase-based regimens) in the remaining 9 cases. Locoregional radiotherapy with a dose of 45-62 Gy was administered to the patients who received radiotherapy.

Univariate survival analysis. All the patients were followed until death or through to April 20, 2015 (deadline date of our study), with a median follow-up of 38 months (range, 1-132 months). The median OS (mOS) was 96 months for all patients, with a 5-year survival rate of $68.0 \%$. However, these data were obviously below average for patients with extranasal primary tumors (63 months, $43.4 \%$ ), ECOG score $\geq 2$ (10 months, 0\%), IPI score 3-5 (9 months, 21.4\%), stage III/IV disease (20 months, 43.6), presence of B symptoms (70 months, $55.7 \%$ ), lymph node involvement (63 months, 57.4\%), and those not receiving radiotherapy (63 months, 55.9\%) (Table I).

The following characteristics were found to indicate poor survival: Primary tumors arising outside the nasal region $(\mathrm{HR}=0.33, \mathrm{P}=0.0079), \mathrm{ECOG}$ score $\geq 2(\mathrm{HR}=0.01, \mathrm{P}<0.0001)$, IPI score 3-5 $(\mathrm{HR}=0.02, \mathrm{P}<0.0001)$, stage III/IV disease $(\mathrm{HR}=0.16, \mathrm{P}<0.0001)$, presence of $\mathrm{B}$ symptoms $(\mathrm{HR}=2.72$, $\mathrm{P}=0.0183)$, lymph node involvement $(\mathrm{HR}=0.34, \mathrm{P}=0.0084)$ and absence of radiotherapy $(\mathrm{HR}=0.30, \mathrm{P}=0.0041$ (Table I).

Comparison between radiotherapy and non-radiotherapy groups. A comparison between the radiotherapy and non-radiotherapy groups by Chi-square tests indicated that patients who received radiotherapy had higher rates of ECOG 0-1 (88.89 vs. $66.67 \%, \mathrm{P}=0.0254)$, IPI 0-2 (91.67 vs. 66.67\%, $\mathrm{P}=0.0099)$, Ann Arbor stage I/II disease ( 75.00 vs. $51.51 \%, \mathrm{P}=0.0426$ ), absence of B symptoms (63.89 vs. 39.39\%, $\mathrm{P}=0.0419)$, normal serum LDH level (63.89 vs. 39.39\%, P=0.0419), and absence of lymph node involvement (63.89 vs. 39.39\%, $\mathrm{P}=0.0419)$ (Table II). Therefore, the survival results of the two groups cannot be compared directly.

Multivariate survival analysis. Cox regression multivariate analysis yielded the following significant prognostic factors for OS in patients with ENKL: ECOG score (HR=9.537, $\mathrm{P}<0.0001)$, disease stage $(\mathrm{HR}=5.500, \mathrm{P}=0.006), \mathrm{IPI}(\mathrm{HR}=3.650$, $\mathrm{P}=0.022)$, presence of $\mathrm{B}$ symptoms $(\mathrm{HR}=3.070, \mathrm{P}=0.039)$ and radiotherapy $(\mathrm{HR}=2.970, \mathrm{P}=0.046)$, indicating that these factors were independently associated with survival of ENKL (Table III).

Analysis of the effect of diverse therapies on survival. To further assess the effect of different regimens, the patients were divided into subgroups by different treatment regimens, different stages and different primary sites. Considering that the radiotherapy group included patients who had received radiotherapy alone as well as chemoradiotherapy, survival was analyzed among the chemoradiotherapy, chemotherapy and radiotherapy groups. The mOS of patients was 118, 63 and 132 months, respectively, and the corresponding 5-year survival rates were $77.5,45.0$ and $83.3 \%$, respectively $(\mathrm{P}=0.0412)$ (Fig. 1A); however, no statistically significant difference was found between the chemoradiotherapy and radiotherapy groups $(\mathrm{HR}=1.534, \mathrm{P}=0.6371)$ (Fig. 1B). These results indicated that the effects were different among the three treatment regimens, but chemoradiotherapy did not 
Table I. Univariate analysis of overall survival by clinical factors $(n=69)$.

\begin{tabular}{|c|c|c|c|c|c|c|c|}
\hline \multirow[b]{2}{*}{ Clinical factors } & \multirow[b]{2}{*}{ No. } & \multicolumn{2}{|c|}{ Survival rate $(\%)$} & \multirow[b]{2}{*}{ mOS (months) } & \multirow[b]{2}{*}{ P-value (log-rank) } & \multirow[b]{2}{*}{ HR } & \multirow[b]{2}{*}{$95 \% \mathrm{CI}$} \\
\hline & & 1-year & 5 -year & & & & \\
\hline \multicolumn{8}{|l|}{ Gender } \\
\hline Men & 50 & 81.9 & 71.1 & 96 & 0.5698 & 1.31 & $0.51-3.37$ \\
\hline Women & 19 & 73.3 & 58.8 & 118 & & & \\
\hline \multicolumn{8}{|l|}{ Age (years) } \\
\hline$\leq 60$ & 52 & 80.7 & 65.4 & 96 & 0.8103 & 1.13 & $0.43-2.96$ \\
\hline$>60$ & 17 & 76.0 & 76.0 & 87 & & & \\
\hline \multicolumn{8}{|l|}{ Primary site } \\
\hline Nasal & 40 & 84.8 & 81.5 & 132 & $0.0079^{\mathrm{a}}$ & 0.33 & $0.14-0.75$ \\
\hline Extranasal & 29 & 72.4 & 43.4 & 63 & & & \\
\hline \multicolumn{8}{|l|}{ ECOG score } \\
\hline $0-1$ & 54 & 92.6 & 83.8 & 132 & $<0.0001^{\mathrm{a}}$ & 0.01 & $0.003-0.03$ \\
\hline$\geq 2$ & 15 & 33.3 & 0 & 10 & & & \\
\hline \multicolumn{8}{|l|}{ IPI } \\
\hline $0-2$ & 55 & 90.8 & 80.2 & 132 & $<0.0001^{\mathrm{a}}$ & 0.02 & $0.006-0.08$ \\
\hline $3-5$ & 14 & 35.7 & 21.4 & 9 & & & \\
\hline \multicolumn{8}{|l|}{ Ann Arbor stage } \\
\hline $\mathrm{I} / \mathrm{II}$ & 44 & 95.5 & 82.5 & 132 & $<0.0001^{\mathrm{a}}$ & 0.16 & $0.06-0.39$ \\
\hline III/IV & 25 & 52.0 & 43.6 & 20 & & & \\
\hline \multicolumn{8}{|l|}{ B symptoms } \\
\hline Yes & 33 & 63.5 & 55.7 & 70 & $0.0183^{\mathrm{a}}$ & 2.72 & $1.19-6.24$ \\
\hline No & 36 & 94.3 & 79.1 & 96 & & & \\
\hline \multicolumn{8}{|c|}{ Serum LDH (U/L) } \\
\hline Normal & 36 & 83.2 & 69.9 & 132 & 0.1861 & 0.58 & $0.26-1.30$ \\
\hline Elevated & 33 & 75.6 & 65.7 & 70 & & & \\
\hline \multicolumn{8}{|c|}{ Bone marrow involvement } \\
\hline Yes & 3 & 66.7 & 66.7 & 132 & 0.8552 & 1.22 & $0.14-10.98$ \\
\hline No & 66 & 80.1 & 68.1 & 96 & & & \\
\hline \multicolumn{8}{|c|}{ Lymph node involvement } \\
\hline Yes & 33 & 66.7 & 57.4 & 63 & $0.0084^{\mathrm{a}}$ & 0.34 & $0.15-0.76$ \\
\hline No & 36 & 91.7 & 75.3 & 132 & & & \\
\hline \multicolumn{8}{|l|}{ Surgery } \\
\hline Yes & 5 & 100.0 & 100.0 & 95 & 0.1413 & 0.33 & $0.08-1.45$ \\
\hline No & 64 & 77.9 & 65.5 & 96 & & & \\
\hline \multicolumn{8}{|l|}{ Radiotherapy } \\
\hline Yes & 36 & 94.3 & 77.9 & 132 & $0.0041^{\mathrm{a}}$ & 0.30 & $0.13-0.68$ \\
\hline No & 33 & 64.7 & 55.9 & 63 & & & \\
\hline \multicolumn{8}{|l|}{ Chemotherapy } \\
\hline Yes & 59 & 81.3 & 69.8 & 96 & 0.5267 & 0.67 & $0.20-2.29$ \\
\hline No & 10 & 70.0 & 58.3 & 132 & & & \\
\hline \multicolumn{8}{|l|}{ HSCT } \\
\hline Yes & 2 & 100.00 & 100.00 & 34 & 0.4089 & 0.35 & $0.03-4.23$ \\
\hline No & 67 & 78.9 & 67.2 & 96 & & & \\
\hline All patients & 69 & 79.6 & 68.0 & 96 & & & \\
\hline
\end{tabular}

${ }^{\text {a }}$ < 0.05 . mOS, median overall survival; HR, hazard ratio; CI, confidence interval; ECOG, Eastern Cooperative Oncology Group; IPI, international prognostic index; LDH, lactate dehydrogenase; HSCT, hematopoietic stem cell transplantation. 
provide a significant advantage over radiotherapy. Since the chemotherapy regimens were mainly anthracycline-based, the patients were divided into the anthracycline-based and non-anthracycline based regimen groups; however, no significant differences were found between these groups in terms of mOS and 5-year survival rates (Table IV).

The survival of patients was then compared by different stages, different primary sites and different treatment regimens. The results revealed a difference between the radiotherapy and non-radiotherapy groups for patients with extranasal-type stage III/IV disease. The mOS for patients with extranasal-type stage III/IV disease in the radiotherapy and non-radiotherapy groups was 81.5 and 12 months, respectively, and the 5-year survival rates differed significantly between the groups (100 vs. 0\%, respectively; $\mathrm{P}=0.0229$ ). No significant differences were found between other groups in terms of mOS and 5-year survival rates. Our data demonstrated that patients with advanced disease may also benefit from radiotherapy (Table IV).

\section{Discussion}

ENKL is a rare extranodal malignancy with poor prognosis. This retrospective study analyzed the clinical characteristics of patients with ENKL. In agreement with previous studies $(2,13)$, our data demonstrated that ECOG score, stage, IPI, presence of B symptoms and treatment with radiotherapy were independently associated with the survival of ENKL. However, the LDH level did not exhibit an independent association with survival, which differed from the results of other studies $(1,8)$, despite the fact that the mOS of patients with elevated serum LDH was below average (70 vs. 96 months) (Table I). A larger sample size is required to further investigate this point.

Radiotherapy is one of the most important treatments for ENKL and has been confirmed in previous studies to improve patient outcomes $(7,8)$. Consistent with these prior reports, our results demonstrated that radiotherapy was able to increase the mOS and 5-year survival rate (132 months, $77.9 \%$ vs. 63 months, $55.9 \%, \mathrm{P}=0.0041$ ) of ENKL (Table I) and was one of the independent prognostic factors for survival (Table III). However, the majority of the studies on ENKL evaluated nasal-type and early-stage disease (14), while few focused on extranasal and late-stage disease. In our study, we also observed that, for patients with stage III/IV disease, the mOS and 5-year survival rate of those receiving radiotherapy were 96 months and $80.0 \%$, respectively, which were more favorable compared with those of patients not receiving radiotherapy (12 months and $30.3 \%$, respectively), although this difference did not quite reach statistical significance with the number of investigated patients $(\mathrm{P}=0.0656)$. In the subgroup analysis stratified by stage and primary site, a statistical difference was observed in the stage III/IV extranasal subgroup between the radiotherapy and non-radiotherapy groups $(\mathrm{P}=0.0229)$, but the samples were relatively small and unbalanced (Table IV). Taken together, our results indicated that patients with advanced disease may also benefit from radiotherapy, although further studies are required to support our findings.

Recently, a meta-analysis of 8 clinical studies compared the efficacy of chemoradiotherapy and chemotherapy for treating early-stage ENKL (10), concluding that chemoradiotherapy
Table II. Comparison of clinical factors between different treatment groups with the Chi-square test.

\begin{tabular}{|c|c|c|c|}
\hline Clinical factors & $\begin{array}{c}\text { Radiotherapy } \\
\mathrm{n}=36(\%)\end{array}$ & $\begin{array}{c}\text { Non-radiotherapy } \\
n=33(\%)\end{array}$ & P-value \\
\hline \multicolumn{4}{|l|}{ Gender } \\
\hline Men & $26(72.22)$ & $24(72.73)$ & 0.9626 \\
\hline Women & $10(27.78)$ & $9(27.27)$ & \\
\hline \multicolumn{4}{|l|}{ Age (years) } \\
\hline$\leq 60$ & $27(75.00)$ & $25(75.76)$ & 0.9418 \\
\hline$>60$ & $9(25.00)$ & $8(24.24)$ & \\
\hline \multicolumn{4}{|l|}{ Primary site } \\
\hline Nasal & $24(66.67)$ & $16(48.48)$ & 0.1264 \\
\hline Extranasal & $12(33.33)$ & $17(51.51)$ & \\
\hline \multicolumn{4}{|l|}{ ECOG score } \\
\hline $0-1$ & $32(88.89)$ & $22(66.67)$ & $0.0254^{\mathrm{a}}$ \\
\hline$\geq 2$ & $4(11.11)$ & $11(33.33)$ & \\
\hline \multicolumn{4}{|l|}{ IPI } \\
\hline $0-2$ & 33 (91.67) & $22(66.67)$ & $0.0099^{\mathrm{a}}$ \\
\hline $3-5$ & $3(8.33)$ & $11(33.33)$ & \\
\hline \multicolumn{4}{|l|}{ Ann Arbor stage } \\
\hline $\mathrm{I} / \mathrm{II}$ & $27(75.00)$ & $17(51.51)$ & $0.0426^{\mathrm{a}}$ \\
\hline III/IV & $9(25.00)$ & $16(48.48)$ & \\
\hline \multicolumn{4}{|l|}{ B symptoms } \\
\hline Yes & $13(36.11)$ & $20(60.60)$ & $0.0419^{\mathrm{a}}$ \\
\hline No & $23(63.89)$ & $13(39.39)$ & \\
\hline \multicolumn{4}{|l|}{$\begin{array}{l}\text { Serum LDH } \\
(\mathrm{U} / \mathrm{l})\end{array}$} \\
\hline Normal & $23(63.89)$ & $13(39.39)$ & $0.0419^{\mathrm{a}}$ \\
\hline Elevated & $13(36.11)$ & $20(60.60)$ & \\
\hline \multicolumn{4}{|l|}{$\begin{array}{l}\text { Bone marrow } \\
\text { involvement }\end{array}$} \\
\hline Yes & $1(2.78)$ & $2(6.06)$ & 0.5042 \\
\hline No & $35(97.22)$ & $31(93.94)$ & \\
\hline \multicolumn{4}{|l|}{$\begin{array}{l}\text { Lymph node } \\
\text { involvement }\end{array}$} \\
\hline Yes & $13(36.11)$ & $20(60.60)$ & $0.0419^{\mathrm{a}}$ \\
\hline No & $23(63.89)$ & $13(39.39)$ & \\
\hline \multicolumn{4}{|l|}{ Surgery } \\
\hline Yes & $4(11.11)$ & $1(3.03)$ & 0.1959 \\
\hline No & $32(88.89)$ & $32(96.97)$ & \\
\hline \multicolumn{4}{|l|}{ Chemotherapy } \\
\hline Yes & $29(72.22)$ & $30(90.91)$ & 0.2223 \\
\hline No & $7(19.44)$ & $3(9.09)$ & \\
\hline \multicolumn{4}{|l|}{ HSCT } \\
\hline Yes & $0(0.0)$ & $2(6.06)$ & 0.1339 \\
\hline No & $36(100.00)$ & $31(93.94)$ & \\
\hline
\end{tabular}

${ }^{\mathrm{a}} \mathrm{P}<0.05$. ECOG, Eastern Cooperative Oncology Group; IPI, international prognostic index; LDH, lactate dehydrogenase; HSCT, hematopoietic stem cell transplantation. 
Table III. Multivariate analysis of survival with Cox proportional hazards model.

$95 \%$ CI for $\operatorname{Exp}(\mathrm{B})$

\begin{tabular}{lccccccrr}
\cline { 5 - 8 } Clinical factors & $\mathrm{B}$ & $\mathrm{SE}$ & Wald & $\mathrm{df}$ & Sig. & Exp (B) & Lower & Upper \\
\hline Age & 0.025 & 0.015 & 2.800 & 1 & 0.094 & 1.026 & 0.996 & 1.057 \\
ECOG PS score & 2.255 & 0.598 & 14.220 & 1 & $0.000^{\mathrm{a}}$ & 9.537 & 2.954 & 30.796 \\
Stage & 1.705 & 0.614 & 7.702 & 1 & $0.006^{\mathrm{a}}$ & 5.500 & 1.650 & 18.334 \\
IPI & 1.295 & 0.564 & 5.276 & 1 & $0.022^{\mathrm{a}}$ & 3.650 & 1.209 & 11.016 \\
B symptoms & 1.122 & 0.545 & 4.240 & 1 & $0.039^{\mathrm{a}}$ & 3.070 & 1.056 & 8.928 \\
Radiotherapy & 1.089 & 0.546 & 3.969 & 1 & $0.046^{\mathrm{a}}$ & 2.970 & 1.018 & 8.667
\end{tabular}

${ }^{\text {a }}<<0.05$. CI, confidence interval; B, coefficient of covariates; SE, standard error; df, degree of freedom; sig, significance; Exp (B), relative risk; ECOG PS, Eastern Cooperative Oncology Group performance status; IPI, international prognostic index.
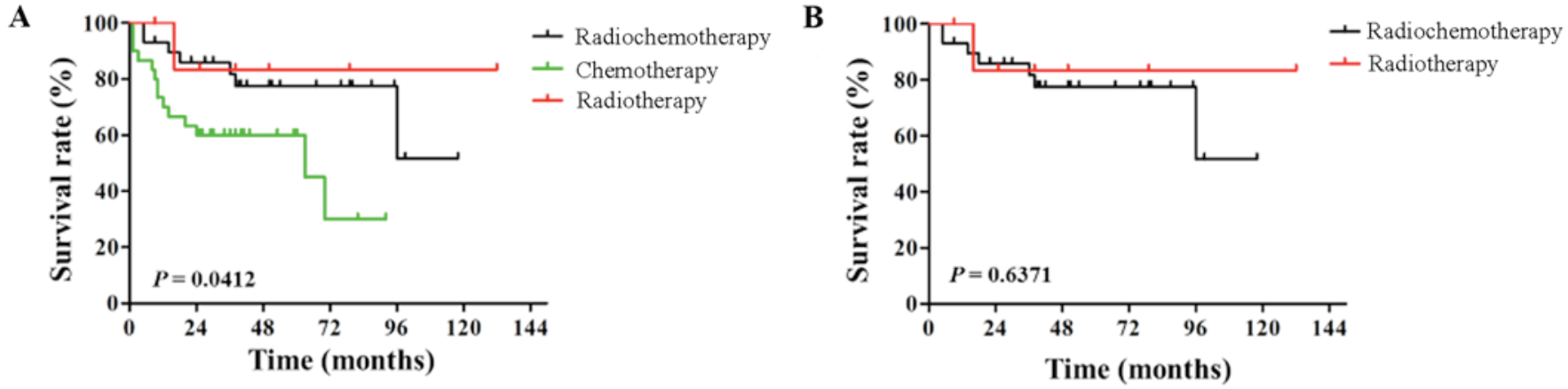

Figure 1. Comparison of overall survival (OS) among patients who received chemoradiotherapy, those who received chemotherapy alone and those who received radiotherapy alone using the Kaplan-Meier method. (A) The survival curves of the chemoradiotherapy vs. chemotherapy vs. radiotherapy groups were significantly different $(\mathrm{P}=0.0412)$. (B) The survival curves of the chemoradiotherapy vs. radiotherapy groups were not significantly different $(\mathrm{P}=0.6371)$.

did not provide a significant advantage over radiotherapy for early-stage nasal-type ENKL. Similarly, we observed that patients in the radiotherapy group achieved a significantly longer mOS and higher 5-year survival rates compared with those in the chemoradiotherapy and the chemotherapy groups (132 months, $83.3 \%$ vs. 118 months, $77.5 \%$ vs. 63 months, $45.0 \%$, respectively; $\mathrm{P}=0.0412$ ) (Fig. 1A). In our study, however, the mOS and 5-year survival rates were not statistically different in the I/II nasal-type subgroups, possibly because the number of patients with stage I/II nasal-type disease was small $(n=31)$. A recent study (15) suggested that, unlike patients with stage I nasal-type disease, who may achieve favorable outcomes when treated with radiotherapy alone, patients with stage II disease or with paranasal involvement should be treated with systemic therapy. Thus, radiotherapy may still be the optimal choice, but whether patients with unfavorable prognosis should be treated with chemotherapy and the optimal chemotherapy regimens require further investigation.

ENKL is often resistant to anthracycline-based chemotherapy (1). Although the prognosis of some patients was improved by non-anthracycline-based chemotherapy, the prognosis of patients with advanced disease remains poor (13). Au et al (16) investigated 136 patients with ENKL and did not find differences between the nasal and extranasal groups in subgroups treated with anthracycline-based chemotherapy, radiotherapy, or a combination of the two. Similarly, in our study, 42 of the 69 patients were administered an anthracycline-based regimen as first-line therapy and the mOS and 5-year survival rates did not differ between those treated with or without radiotherapy.

Obama (17) reported that, for radiotherapy-resistant disease, chemoradiotherapy, including the topoisomerase inhibitors etoposide (VP-16) and irinotecan (CPT-11), may be a therapeutic option. The present study included only 6 cases treated with ECHOP, among which 5 received chemoradiotherapy; thus, more data are required to evaluate this type of treatment. SMILE has been reported as a potential first-line chemotherapy regimen for ENKL patients $(5,18,19)$. A phase II study (20) also demonstrated that SMILE chemotherapy may be a choice for patients with stage IV ENKL with relapsed or refractory disease. The present study included 4 patients treated with the SMILE chemotherapy regimen, but only 1 received sequential radiotherapy. That patient received only 2 cycles of chemotherapy, due to myelosuppression. The SMILE regimen may achieve better outcomes for ENKL, but it is associated with a high risk of myelosuppression during the long treatment period. Furthermore, its clinical value and ability to be used in combination with radiotherapy remain uncertain.

HSCT is a potential therapeutic option, allowing some patients to achieve remission (21). The present study included only 2 patients who had been treated with HSCT, and they have achieved 30 and 34 months of remission. However, further 


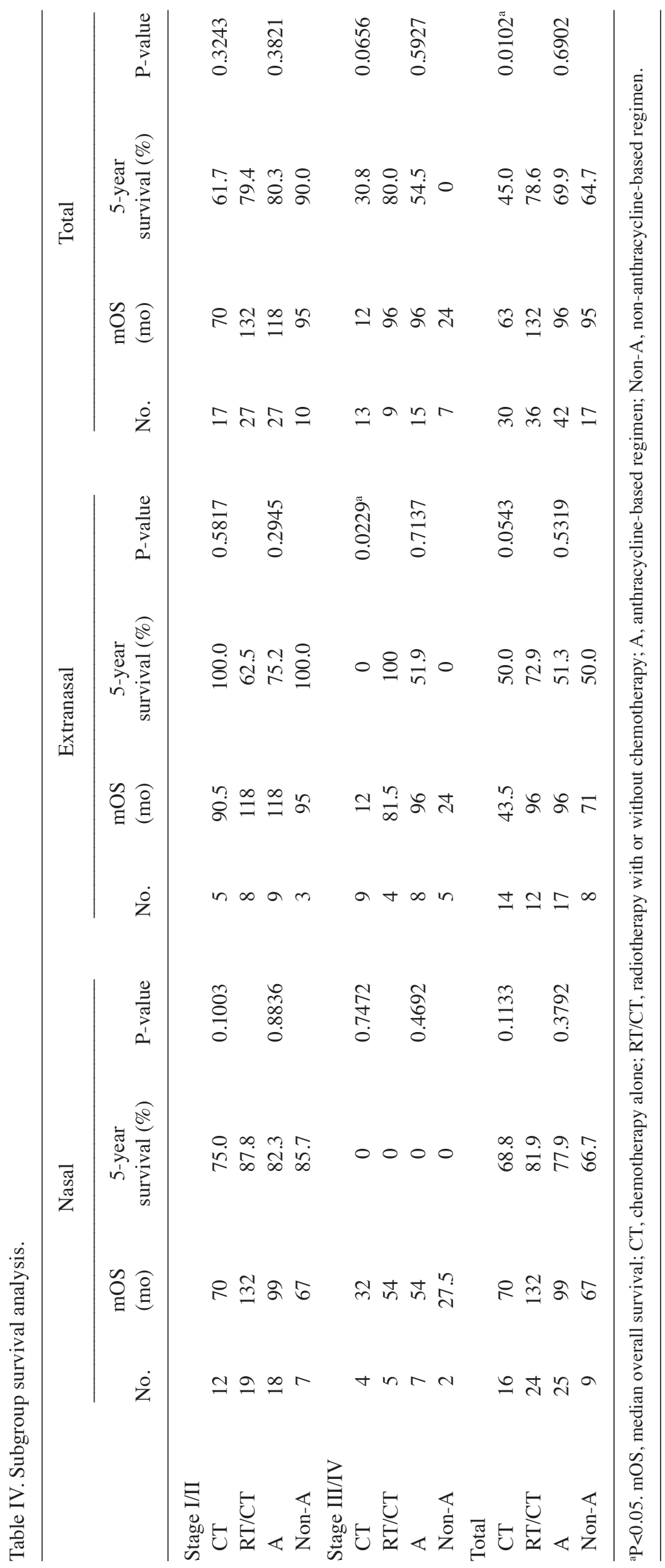


investigation is required to identify more effective therapeutic options for this disease.

In conclusion, ECOG PS score, disease stage, IPI, presence of $\mathrm{B}$ symptoms and treatment with radiotherapy were the independent prognostic factors of OS in patients with ENKL. Radiotherapy achieved better outcomes, but no difference was observed between chemoradiotherapy and radiotherapy. Patients with advanced disease may also benefit from radiotherapy.

\section{Acknowledgements}

The present study was supported by the Natural Science Foundation of China (grant no. 81172169).

\section{References}

1. William BM and Armitage JO: International analysis of the frequency and outcomes of NK/T-cell lymphomas. Best Pract Res Clin Haematol 26: 23-32, 2013.

2. Zhang J, Wang WD, Geng QR, Wang L, Chen XQ, Liu CC and Lv Y: Serum levels of interleukin-9 correlate with negative prognostic factors in extranodal NK/T-cell lymphoma. PloS One 9 : e94637, 2014.

3. Sun J, Yang Q, Lu Z, He M, Gao L, Zhu M, Sun L, Wei L, Li M, Liu C, et al: Distribution of lymphoid neoplasms in China: Analysis of 4,638 cases according to the World Health Organization classification. Am J Clin Pathol 138: 429-434, 2012.

4. Lee J, Suh C, Park YH, Ko YH, Bang SM, Lee JH, Lee DH, Huh J, Oh SY, Kwon HC, et al: Extranodal natural killer T-cell lymphoma, nasal-type: A prognostic model from a retrospective multicenter study. J Clin Oncol 24: 612-618, 2006.

5. Jo JC, Yoon DH, Kim S, Lee BJ, Jang YJ, Park CS, Huh J, Lee SW, Ryu JS and Suh C: Clinical features and prognostic model for extranasal NK/T-cell lymphoma. Eur J Haematol 89: 103-110, 2012.

6. Li X, Liao WH, Zhou JH, Liu BA, Wen JF and Hu ZL: NK/T cell lymphoma involving mediastinum: Report of a case and review of literature. Int J Clin Exp Pathol 7: 6399-6402, 2014.

7. Wang ZY, Liu QF, Wang H, Jin J, Wang WH, Wang SL, Song YW, Liu YP, Fang H, Ren H, et al: Clinical implications of plasma Epstein-Barr virus DNA in early-stage extranodal nasal-type NK/T-cell lymphoma patients receiving primary radiotherapy. Blood 120: 2003-2010, 2012.

8. Chauchet A, Michallet AS, Berger F, Bedgedjian I, Deconinck E, Sebban C, Antal D, Orfeuvre H, Corront B, Petrella T, et al: Complete remission after first-line radio-chemotherapy as predictor of survival in extranodal NK/T cell lymphoma. J Hematol Oncol 5: 27, 2012.

9. Ma HH, Qian LT, Pan HF, Yang L, Zhang HY, Wang ZH, Ma J, Zhao YF, Gao J and Wu AD: Treatment outcome of radiotherapy alone versus radiochemotherapy in early stage nasal natural killer/T-cell lymphoma. Med Oncol 27: 798-806, 2010.
10. Zhang B, Zhu F, Liu X, Zhao J, Li M, Li Y, Huang J, Zou L and Chang Q: Radiotherapy combined with chemotherapy versus radiotherapy alone for early stage nasal natural killer/T-cell lymphoma: A meta-analysis. J Chemother 28: 65-71, 2016.

11. National Comprehensive Cancer Network: NCCN Clinical Practice Guidelines in Oncology: Non-Hodgkin's Lymphomas, Version 2, 2015 https://www.nccn.org/about/nhl.pdf. Accessed July 13, 2015.

12. Swerdlow SH, Campo E, Harris NL, Jaffe ES, Pileri SA, Stein H, Thiele J and Vardiman JW: WHO classification of tumours of haematopoietic and lymphoid tissues. Vol 2, 4th edition, World Health Organization, 2008.

13. Kim SJ, Hong M, Do IG, Lee SH, Ryu KJ, Yoo HY, Hong JY, Ko YH and Kim WS: Serum survivin and vascular endothelial growth factor in extranodal NK/T-cell lymphoma, nasal type: Implications for a potential new prognostic indicator. Haematologica 100: e106-e109, 2015.

14. Li YX, Wang H, Jin J, Wang WH, Liu QF, Song YW, Wang ZY, Qi SN, Wang SL, Liu YP, et al: Radiotherapy alone with curative intent in patients with stage I extranodal nasal-type NK/T-cell lymphoma. Int J Radiat Oncol Biol Phys 82: 1809-1815, 2012.

15. Li YX, Liu QF, Wang WH, Jin J, Song YW, Wang SL, Liu YP, Liu XF, Zhou LQ and Yu ZH: Failure patterns and clinical implications in early stage nasal natural killer/T-cell lymphoma treated with primary radiotherapy. Cancer 117: 5203-5211, 2011.

16. Au WY, Weisenburger DD, Intragumtornchai T, Nakamura S, Kim WS, Sng I, Vose J, Armitage JO and Liang R; International Peripheral T-Cell Lymphoma Project: Clinical differences between nasal and extranasal natural killer/T-cell lymphoma: A study of 136 cases from the International Peripheral T-Cell Lymphoma Project. Blood 113: 3931-3937, 2009.

17. Obama K: Concomitant use of radiotherapy and two topoisomerase inhibitors to treat adult T-cell leukemia with a radiotherapy-resistant bulky disease: A case series. Am J Blood Res 4: 106-109, 2014.

18. Kwong YL, Kim WS, Lim ST, Kim SJ, Tang T, Tse E, Leung AY and Chim CS: SMILE for natural killer/T-cell lymphoma: Analysis of safety and efficacy from the Asia Lymphoma Study Group. Blood 120: 2973-2980, 2012.

19. Lim YL, Pack HS, Park JE, Oh JR and Kong JH: Extranodal natural killer/T-cell lymphoma of the tenosynovium of the hand. Korean J Intern Med 30: 122-124, 2015.

20. Yamaguchi M, Kwong YL, Kim WS, Maeda Y, Hashimoto C, Suh C, Izutsu K, Ishida F, Isobe Y, Sueoka E, et al: Phase II study of SMILE chemotherapy for newly diagnosed stage IV, relapsed, or refractory extranodal natural killer (NK)/T-cell lymphoma, nasal type: The NK-Cell Tumor Study Group study. J Clin Oncol 29: 4410-4416, 2011

21. LaPorte J, Morris L and Koepke J: Long complete remission achieved with the combination therapy of Cisplatin and gemcitabine in a patient with aggressive natural killer cell leukemia. Case Rep Hematol 2015: 715615, 2015. 